\title{
Correspondence:
}

\section{Disease severity and time to diagnosis in 100 patients with newly diagnosed disease modifying antirheumatic drugs treatment-naïve rheumatoid arthritis}

Rheumatoid arthritis (RA) is a chronic inflammatory disease characterized by joint swelling, joint tenderness, and destruction of synovial joints, leading to severe disability and premature mortality. ${ }^{1}$ However, advances in understanding the pathogenesis of the disease have resulted in the emergence of new therapeutic options with improved outcomes. Presently, aggressive treatment is initiated soon after diagnosis and treatment is escalated to achieve clinical remission. However, sparse objective data are available in PUBMED indexed journals regarding the symptom duration at the time of diagnosis and disease severity in patients with RA from Andhra Pradesh. We, therefore, studied the clinical presentation, time-lapse from onset of symptoms to diagnosis, disease severity and pattern of laboratory investigations in patients with disease modifying antirheumatic drugs (DMARDs)treatment-naïve newly diagnosed RA.

This is a cross-sectional study was conducted at our tertiary care hospital, Sri Venkateswara Institute of Medical Sciences, Tirupati during the period June 2012 to May 2013. RA was diagnosed as per the American College of Rheumatology (ARA)/European League Against Rheumatism (EULAR) 2010 criteria. $^{2}$ One hundred patients who were newly diagnosed to have RA were included in the study. Eligible patients were at least 18 years of age and were DMARDS treatment naïve. Patients under 18 years of age, those with autoimmune diseases other than rheumatoid arthritis, overlap syndrome, active infection, pregnancy and patients unwilling to participate in the study were excluded. The study was approved by the institutional Research Approval Committee and Ethics Committee. Written informed consent was obtained from all the patients participating in the study.

A detailed history was taken from the patient regarding the presentation, duration of symptoms, any other comorbid illnesses and information regarding the disease-specific variables, such as, duration of morning stiffness, tender and swollen joints, deformities, disability associated with it. Baseline evaluation included demographic information, disease activity score including 28 joints (DAS28) evaluating the number of swollen joints, number of tender joints, the patients' global assessment of health measured on a visual analogue scale (VAS, range 0-100 mm), and erythrocyte sedimentation rate $(\mathrm{ESR}) .^{3}$ complete haemogram and antero-posterior radiographs of both hands. A score of DAS28 between 2.6-3.2 indicates low disease activity, >3.2- $\leq 5.1$ moderate and $>5.1$ high disease activity. ${ }^{3}$

Rheumatoid factor (RF) was measured by latex agglutination test (Biosystems S.A., Barcelona). Anti-cyclic citrullinated protein (anti-CCP) antibodies were measured using an enzyme linked immunosorbent assay (ELISA) (Biosystems S.A., Barcelona); a titre of greater than $10 \mathrm{U} / \mathrm{mL}$ considered as positive. $\mathrm{C}$-reactive protein (CRP) was estimated with latex agglutination test (CRP latext Test Kit, Plasmatec, Dorset). ESR was estimated by Westergen's method. Descriptive statistics were expressed as mean and standard deviation for continuous variables that were normally distributed, median [interquartile range (IQR)] for other variables, numbers and percentages for qualitative variables. Independent sample t-test was used to compare DAS28 between anti-CCP positive and anti-CCP negative groups. DAS28 was compared across various strata of CRP using chi-square test with Yate's correction.

Their mean age was $48.9 \pm 11.9$ years; there were 84 females. Patients had median (IQR) symptom duration of 5 (4-7) months. Salient laboratory abnormalities included thrombocytosis ( $90 \%$ ); lymphocytosis (81\%); neutrophilia (30\%); anaemia (18\%); and leukocytosis (10\%). The median (IQR) ESR ( $\mathrm{mm}$ at the end of first hour) at presentation was 40 (22.5-79.5); an ESR greater than 100 was found in $15 \%$ patients and ESR less than 
5 was observed in 7\% patients. Anti-CCP antibody and RF were positive in $38 \%$ and $58 \%$ patients respectively. Both tests were negative in $29 \%$ patients. Anti-CCP antibody tested positive in 25 $(43.1 \%)$ of patients with a positive $\mathrm{RF}(\mathrm{n}=58)$ and in $13(31 \%)$ of those with a negative RF test result $(n=42)$. As per DAS28, $41 \%$ patients presented with severe disease; $36 \%$ presented with moderate disease, while $23 \%$ presented with a mild disease. Mean DAS28 was higher in patients who were anti-CCP antibody positive compared with those who were anti-CCP antibody negative $(5.029 \pm 1.471$ Vs $4.333 \pm 1.366 ; 0.017)$ suggesting that anti-CCP antibody positive patients presented with a more severe disease. Comparison of DAS28 score across various CRP levels is shown in Table 1. The CRP levels were signifi-

Table 1: Comparison of CRPlevels in patients with varying degrees of disease severity

\begin{tabular}{llcll}
\hline $\begin{array}{l}\text { Disease } \\
\text { severity } \\
\text { (DAS28) }\end{array}$ & \multicolumn{5}{c}{$\begin{array}{c}\text { CRP levels } \\
\text { (mg/dL) }\end{array}$} \\
\hline Mild (<3.2) & 12 & 7 & 3 & 1 \\
Moderate (3.2-5.1) & 3 & 20 & 11 & 2 \\
Severe (>5.1) & 1 & 3 & 10 & 27 \\
\hline
\end{tabular}

DAS28=disease activity score including 28 joints; $\mathrm{CRP}=\mathrm{C}$-reactive protein cantly higher $(>24 \mathrm{mg} / \mathrm{dL})$ in patients with severe disease $(\mathrm{DAS} 28>5.1)\left(\chi^{2}=71.297 \mathrm{p}=0.000\right)$.

In the present study, women in the fifth decade of life were predominantly affected. Median symptom duration of 5 months and majority (77\%) of the patients presented with moderate or severe disease suggesting that RA is being diagnosed late In its course. Our findings suggest that there is a need to educate primary care physicians regarding the approach for early diagnosis of RA This is important considering the fact that early institution of specific therapy can be effective in limiting disability and minimizing the complications.

\section{REFERENCES}

1. Scott DL, Wolfe F, Huizinga TW. Rheumatoid arthritis. Lancet 2010;376:1094-108.

2. Aletaha D, Neogi T, Silman AJ, Funovits J, Felson DT, Bingham CO, et al. 2010 Rheumatoid arthritis classification criteria: an American College of Rheumatology/European League Against Rheumatism collaborative initiative. Arthritis Rheum 2010;62:2569-81.

3. Prevoo ML, van' t Hof MA, Kuper HH, van Leeuwen MA, van de Putte LB, van Riel PL. Modified disease activity scores that include twenty-eight-joint counts. Development and validation in a prospective longitudinal study of patients with rheumatoid arthritis. Arthritis Rheum 1995;38:44-8. 